\title{
A humanized model of microvascular infection
}
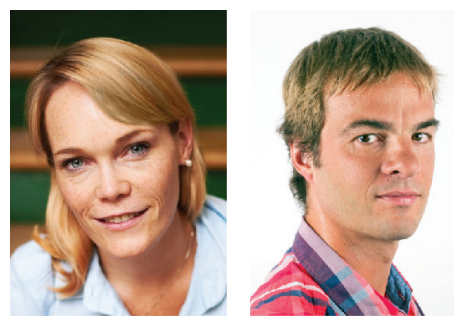

\author{
"Understanding how \\ Neisseria meningitidis causes these \\ vascular injuries during septic shock \\ requires an animal model to mimic this \\ form of the infection."
}

Keira Melican ${ }^{1,2} \&$ Guillaume Duménil*1,2

IINSERM U970, Paris Cardiovascular Research Centre, Paris, F-75015, France

ZUniversité Paris Descartes, Faculté de Médecine Paris Descartes, Paris, F-75006 France

*Author for correspondence: Tel.: +33 153988049 = Fax: +33 153987953 = guillaume.dumenil@inserm.fr

Animal models are a cornerstone of infectious disease research, invaluable to investigating how the infectious process progresses and damages the host tissues. Their use has led to great advances in research, treatment and prevention. However, a number of pathogens are human specific, limiting the use of animal models. These pathogens include HIV, HBV, parasites such as the malariacausing Plasmodium falciparum and bacterial pathogens including Haemophilus influenzae, Listeria monocytogenes, Salmonella typhi and Neisseria meningitidis. These infections represent a serious socioeconomic problem worldwide and are the focus of much ongoing research.

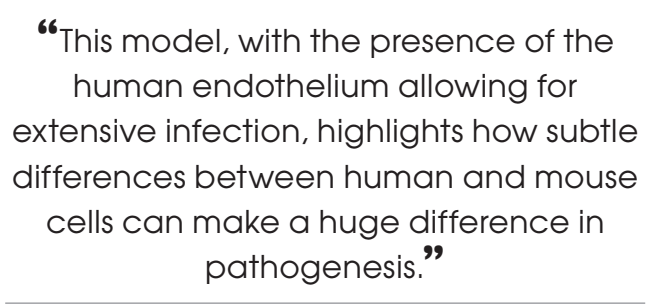

The human tropism of these pathogens depends on specific interactions between the host and pathogen and as our knowledge increases, the number of these species-specific steps is growing. A classic example is the interaction between an adhesin and its host receptor, such as in the case of internalin-A and E-cadherin for L. monocytogenes [1]. Interactions with the immune system can also generate species specificity. Infection with $S$. typhi provides an interesting illustration. Unlike humans, mice express TLR11, which provides protection against S. typhi infection. TLR11-knockout mice become as susceptible to the infection as humans [2]. In the case of $N$. meningitidis, our pathogen of interest, several steps of the infection process have been identified as human-specific. First and foremost, type IV pili (Tfp)-dependent adhesion is species-specific; secondly, iron scavenging by $N$. meningitidis requires human transferrin [3] and thirdly, the complement evasion pathway is human-specific [4].

Research teams over the world have encountered this issue of species specificity and there has been a significant push towards the development of 'humanized' models for these infections. Rodent models hold a significant cost and accessibility advantage over models such as primates, which have historically been used in the study of HIV, for example [5].

Humanized rodent models that are currently in use in infectious disease research include the human immune system (HIS) mice, which are created by introducing either human hematopoietic progenitor cells or human peripheral blood cells into immunodeficient mice. HIS mice of various types have been used to study infection with HIV [6] as well as bacterial pathogens such as $S$. typhi [7]. Transgenic mice engineered to express human proteins have proven useful when the factor involved in pathogen interaction is known (e.g., E-cadherin to study invasion by L. monocytogenes) [8]. Another approach involves the grafting of human tissue onto immunodeficient mice. Human liver chimeric mice, made by transplanting human hepatocytes, have been used for hepatic pathogens such as the hepatitis viruses and the malaria parasite $P$. falciparum $[9,10]$. Transplantation of fetal human intestine under the skin in immunodeficient mice has been used to study a number of enteropathic pathogens including Salmonella [11], Shigella [12] and enterohemorrhagic and enteroaggregative Escherichia coli $[13,14]$.

Developing an animal model for $N$. meningitidis infection raises some specific difficulties. For instance, N. meningitidis is responsible for two clinically different diseases that need to be addressed separately. $N$. meningitidis causes septic

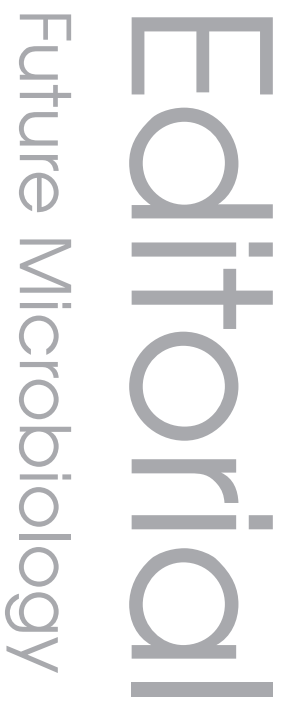

\section{Keywords}

- humanized models

- Neisseria meningitidis

- Purpura fulminans = sepsis Future 
shock and meningitis, which can occur either separately or concomitantly. While meningitis is the more well-known of these two diseases, meningococcal sepsis is more deadly, with a fatality rate of up to $52 \%$, and is responsible for $90 \%$ of fatal cases [15]. The nonblanching petechial and purpuric rashes that appear on the skin of the patient are hallmarks of meningococcal sepsis and are believed to be the result of extensive damage to the vasculature in the skin. Histological and pathological data collected from the dermal lesions of patients show bacterial aggregates closely associated with capillaries. Infection is linked to activation of the clotting cascade, thrombosis, congestion and stasis within the microvessels $[16,17]$. Endothelial cell breakdown, vascular leakage and infiltration of immune cells into the area are also observed $[15,18,19]$. Understanding how $N$. meningitidis causes these vascular injuries during septic shock requires an animal model to mimic this form of the infection.

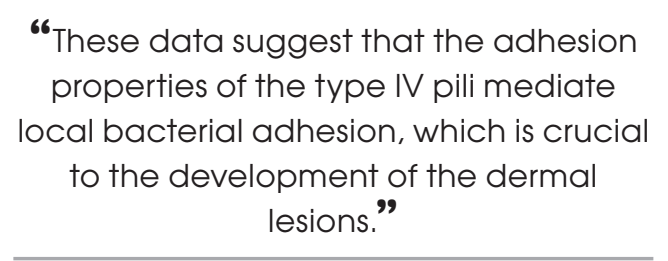

Although Tfp-dependent adhesion is generally thought to be an important step in the infection process, the cellular receptor for this bacterial organelle remains to be identified and, at this stage, a knock-in strategy cannot be envisioned. As an alternative, an approach based on tissue grafting was chosen. Human skin, containing an abundance of dermal capillaries, is grafted onto immunodeficient mice [20]. This model was initially developed to study graft rejection [20]. This results in functional human microvessels connected to, and perfused with, the mouse circulation. $N$. meningitidis introduced intravenously into this model becomes rapidly and exclusively associated with the human skin graft. Closer inspection showed that bacteria could be found within the human capillaries [21]. No bacteria were found in similar sized mouse vessels in the local area. This model, with the presence of the human endothelium allowing for extensive infection, highlights how subtle differences between human and mouse cells can make a huge difference in pathogenesis.

Areas that contained bacteria also showed immune cell infiltration and extensive vascular damage, with thrombosis, congestion, endothelial damage and vascular leak. Approximately
$30 \%$ of infections resulted in visible purpura on the grafted skin. This pathology closely mimicked that seen in clinical patients and represents the first time we have been able to model the development of the purpuric rash. The chimeric nature of the model allowed for the the detection of human cytokine upregulation, specifically IL- 6 and -8 , which was associated with the infected endothelium [21].

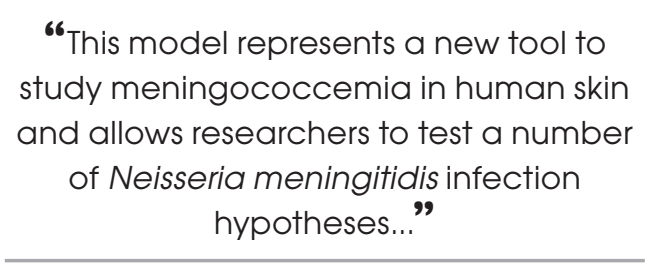

The role of Tfp in the development of the dermal lesions was also investigated. A mutant lacking Tfp showed no accumulation in the human skin graft, no significant inflammatory or vascular pathology and no identifiable human cytokine signaling. These data suggest that the adhesion properties of the Tfp mediate local bacterial adhesion, which is crucial to the development of the dermal lesions. It has previously been thought that circulating bacteria and endotoxin led to the extensive microvasculature damage seen in meningococcal disease. These findings are important to the understanding of the disease mechanisms, especially because they establish a strong link between bacterial adhesion and vascular damage.

This model represents a new tool to study meningococcemia in human skin and allows researchers to test a number of $N$. meningitidis infection hypotheses, previously shown in vitro, in an in vivo setting. This model is malleable from both the host and pathogen aspects, allowing flexibility in terms of both bacterial strains and the environment into which they are introduced. For instance, an extensive library of $N$. meningitidis mutants has been described, some with well-defined in vitro phenotypes that would be of great interest to test in vivo [22]. On the host side, murine immune cell populations and vascular components can be manipulated with antibodies and pharmacological agents, while the immunodeficient background would allow for the allogenic transfer of human immune cell populations. This concept of combining 'humanized' models (i.e., human tissue and HIS) has been previously suggested for the liver chimeras and could also be applied to the skin model [5].

In terms of clinical applications, using this model to test ways to pharmacologically block 
bacterial-host interactions could prove to be an exciting new treatment option for meningococcemia. Interfering with adhesion and preventing the development of the dermal lesions could lead to far better outcomes in terms of tissue necrosis, debridement and amputations, which are common in meningococcemia survivors. Another exciting prospect is to use these new humanized models to test the efficiency of vaccine targets. Currently the lack of in vivo models for meningococcus means there is no preclinical animal model for new vaccine candidates. Although the mouse model used in the skin graft model is immunodeficient, it would be possible to use antibodies generated in immunocompetent mice to test their bactericidal activities, especially their activity on adherent microcolonies in the vessels.

Furthermore, it would now be of interest to extend these findings beyond $N$. meningitidis and investigate the importance of local endothelial adhesion in other bloodborne infections. The role bacterial adhesion plays in vascular damage and purpuric rash development in other sepsis-causing pathogens can now be addressed. Therefore, this skin graft approach adds to the toolbox for researchers studying deadly infections targeting the human vasculature.

\section{Financial \& competing interests disclosure \\ The authors have no relevant affiliations or financial involvement with any organization or entity with a financial interest in or financial conflict with the subject matter or materials discussed in the manu- script. This includes employment, consultancies, honoraria, stock ownership or options, expert testi- mony, grants or patents received or pending, or royalties. \\ No writing assistance was utilized in the production of this manuscript.}

\section{References}

1. Disson $\mathrm{O}$, Grayo S, Huillet $\mathrm{E}$ et al. Conjugated action of two species-specific invasion proteins for fetoplacental listeriosis. Nature 455(7216), 1114-1118 (2008).

2. Mathur R, Oh H, Zhang D et al. A mouse model of Salmonella typhi infection. Cell 151(3), 590-602 (2012).

3. Calmettes C, Alcantara J, Yu RH, Schryvers $\mathrm{AB}$, Moraes TF. The structural basis of transferrin sequestration by transferrinbinding protein B. Nat. Struct. Mol. Biol. 19(3), 358-360 (2012).

4. Kugelberg E, Gollan B, Tang CM. Mechanisms in Neisseria meningitidis for resistance against complement-mediated killing. Vaccine 26(Suppl. 8), I34-I39 (2008).

5. Legrand N, Ploss A, Balling R et al. Humanized mice for modeling human infectious disease: challenges, progress, and outlook. Cell Host Microbe 6(1), 5-9 (2009).

6. Ito M, Hiramatsu H, Kobayashi K et al. NOD/SCID/gamma(c) $)^{\text {null }}$ mouse: an excellent recipient mouse model for engraftment of human cells. Blood 100(9), 3175-3182 (2002).

7. Firoz Mian M, Pek EA, Chenoweth MJ, Ashkar AA. Humanized mice are susceptible to Salmonella typhi infection. Cell. Mol. Immunol. 8(1), 83-87 (2011).

8. Lecuit M, Vandormael-Pournin S, Lefort J et al. A transgenic model for listeriosis: role of internalin in crossing the intestinal barrier. Science 292(5522), 1722-1725 (2001).
9. Mercer DF, Schiller DE, Elliott JF et al. Hepatitis $\mathrm{C}$ virus replication in mice with chimeric human livers. Nat. Med. 7(8), 927-933 (2001).

10. Morosan S, Hez-Deroubaix S, Lunel F et al. Liver-stage development of Plasmodium falciparum, in a humanized mouse model. J. Infect. Dis. 193(7), 996-1004 (2006).

11. Bertelsen LS, Paesold G, Eckmann L, Barrett KE. Salmonella infection induces a hypersecretory phenotype in human intestinal xenografts by inducing cyclooxygenase 2 . Infect. Immun. 71(4), 2102-2109 (2003).

12. Zhang Z, Jin L, Champion G, Seydel KB, Stanley SL Jr. Shigella infection in a SCID mouse-human intestinal xenograft model: role for neutrophils in containing bacterial dissemination in human intestine. Infect. Immun. 69(5), 3240-3247 (2001).

13. Boll EJ, Struve C, Sander A et al. The fimbriae of enteroaggregative Escherichia coli induce epithelial inflammation in vitro and in a human intestinal xenograft model. J. Infect. Dis. 206(5), 714-722 (2012).

14. Golan L, Gonen E, Yagel S, Rosenshine I, Shpigel NY. Enterohemorrhagic Escherichia coli induce attaching and effacing lesions and hemorrhagic colitis in human and bovine intestinal xenograft models. Dis. Model Mech. 4(1), 86-94 (2011).

15. Brandtzaeg P, van Deuren M. Classification and pathogenesis of meningococcal infections. Methods Mol. Biol. 799, 21-35 (2012).

16. Guarner J, Greer PW, Whitney A et al. Pathogenesis and diagnosis of human meningococcal disease using immunohistochemical and PCR assays. Am. J. Clin. Pathol. 122(5), 754-764 (2004).

17. Mairey E, Genovesio A, Donnadieu E et al. Cerebral microcirculation shear stress levels determine Neisseria meningitidis attachment sites along the blood-brain barrier. J. Exp. Med. 203(8), 1939-1950 (2006).

18. Faust SN, Levin M, Harrison OB et al. Dysfunction of endothelial protein C activation in severe meningococcal sepsis. N. Engl. J. Med. 345(6), 408-416 (2001).

19. Harrison $\mathrm{OB}$, Robertson $\mathrm{BD}$, Faust $\mathrm{SN}$ et al. Analysis of pathogen-host cell interactions in purpura fulminans: expression of capsule, type IV pili, and PorA by Neisseria meningitidis in vivo. Infect. Immun. 70(9), 5193-5201 (2002).

20. Murray AG, Petzelbauer P, Hughes CC, Costa J, Askenase P, Pober JS. Human T-cellmediated destruction of allogeneic dermal microvessels in a severe combined immunodeficient mouse. Proc. Natl Acad. Sci. USA 91(19), 9146-9150 (1994).

21. Melican K, Michea Veloso P, Martin T, Bruneval P, Dumenil G. Adhesion of Neisseria meningitidis to dermal vessels leads to local vascular damage and purpura in a humanized mouse model. PLoS Pathog. 9(1), e1003139 (2013).

22. Rusniok C, Vallenet D, Floquet $S$ et al. NeMeSys: a biological resource for narrowing the gap between sequence and function in the human pathogen Neisseria meningitidis. Genome Biol. 10(10), R110 (2009). 